\title{
Double-pulse planar-LIF investigations using fluorescence motion analysis for mixture formation investigation
}

\author{
J. Scholz $\cdot$ T. Wiersbinski $\cdot$ P. Ruhnau • \\ D. Kondermann $\cdot$ C. S. Garbe $\cdot$ R. Hain $\cdot$ \\ V. Beushausen
}

Received: 18 November 2007 /Revised: 8 June 2008/Accepted: 28 June 2008/Published online: 15 August 2008

(C) Springer-Verlag 2008

\begin{abstract}
A concept for dynamic mixture formation investigations of fuel/air mixtures is presented which can equally be applied to several other laser induced fluorescence (LIF) applications. Double-pulse LIF imaging was used to gain insight into dynamic mixture formation processes. The setup consists of a modified standard PIV setup. The "fuel/air ratio measurement by laser induced fluorescence (FARLIF)" approach is used for a quantification of the LIF images in order to obtain pairs of 2D fuel/air ratio maps. Two different evaluation concepts for LIF double pulse images are discussed. The first is based on the calculation of the temporal derivative field of the fuel/air ratio distribution. The result gives insight into the dynamic mixing process, showing where and how the mixture is changing locally. The second concept uses optical flow methods in order to estimate the motion of fluorescence (i.e., mixture) structures to
\end{abstract}

J. Scholz $(\bowtie) \cdot T$. Wiersbinski · V. Beushausen

Department of Photonic Sensor Technology,

Laser-Laboratorium Goettingen e.V.,

Goettingen, Germany

e-mail: Jochen.Scholz@1lg-ev.de; jochen-scholz@gmx.de

V. Beushausen

e-mail: Volker.Beushausen@1lg-ev.de

P. Ruhnau

Computer Vision, Graphics and Pattern Recognition Group,

University of Mannheim, Mannheim, Germany

D. Kondermann · C. S. Garbe

Interdisciplinary Center for Scientific Computing,

University of Heidelberg, Heidelberg, Germany

\section{R. Hain}

Institute of Fluid Mechanics,

Technical University Braunschweig,

Braunschweig, Germany gain insight into the dynamics, showing the distortion and the motion of the inhomogeneous mixture field. For this "fluorescence motion analysis" (FMA) two different evaluation approaches-the "variational gradient based approach" and the "variational cross correlation based approach" - are presented. For the validation of both, synthetic LIF image pairs with predefined motion fields were generated. Both methods were applied and the results compared with the known original motion field. This validation shows that FMA yields reliable results even for image pairs with low signal/noise ratio. Here, the "variational gradient based approach" turned out to be the better choice so far. Finally, the experimental combination of doublepulse FARLIF imaging with FMA and simultaneous PIV measurement is demonstrated. The comparison of the FMA motion field and the flow velocity field captured by PIV shows that both results basically reflect complementary information of the flow field. It is shown that the motion field of the fluorescence structures does not (necessarily) need to represent the actual flow velocity and that the flow velocity field alone can not illustrate the structure motion in any case. Therefore, the simultaneous measurement of both gives the deepest insight into the dynamic mixture formation process. The examined concepts and evaluation approaches of this paper can easily be adapted to various other planar LIF methods (with the LIF signal representing, e.g., species concentration, temperature, density etc.) broadening the insight for a wide range of different dynamic processes.

\section{Introduction}

For the enhancement of many technical processes such as reacting flows, mixing in chemical reactors or mixture 
formation in combustion engines, the knowledge of spatial distributions of molecular species is of great importance. The planar laser-induced fluorescence (PLIF) technique is a well established method to measure two-dimensional maps of concentration or mixture-ratios in a specific plane in the volume of interest. In our recent work we were able to validate a PLIF technique for the quantitative detection of fuel/air ratios (FARLIF) which will be used for mixtureformation investigations in optical engines (Scholz et al. 2006, 2007). In many applications - such as mixture-formation in combustion engines-not only the actual species or mixture distribution is of interest. The temporal evolution (i.e the dynamics) of the species distribution often is the key for understanding and improvement of the underlying fluid-dynamic processes.

Most recent developments on the laser- and cameramarket rendered high-speed LIF imaging accessible for specific applications (e.g., Smith and Sick 2006). Yet, there are still limitations to be overcome. One is that high speed LIF equipment consisting of a high-power high-speed laser and an image-intensified high-speed camera usually is expensive. Others are the limitation in the frame rate (usually in the order of several $\mathrm{kHz}$ ) and the limitation of the excitation wavelength (the shortest wavelength that is commercially available with sufficient output for highspeed LIF is $355 \mathrm{~nm}$, in particular there are no systems available with the excitation wavelength $266 \mathrm{~nm}$ which is often used in LIF applications).

Therefore, we use double-pulse LIF imaging in order to detect the change of fluorescence structures in the mixtureformation process of fuel and air in a test chamber (doublepulse FARLIF). This system operates at $266 \mathrm{~nm}$ excitation and is based on a typical PIV-system (two frequency doubled Nd:YAG lasers at $532 \mathrm{~nm}$ and a PCO dual-frame camera) using additional frequency doublers (to convert the wavelength to $266 \mathrm{~nm}$ ) and an additional adaptable image intensifier. Therefore, such a LIF-system is readily available to many labs owning standard PIV systems.

A look at a LIF double image itself gives an impression of the dynamics, e.g., of a mixing situation or a reacting flow. However, in some cases it is essential to find a more quantitative measure for the change between two images. To this end, two different evaluations of double-pulse LIF images were examined. The first results in the two-dimensional temporal derivative of the quantity corresponding to the LIF signal, in our case the fuel/air-ratio. The second measures the motion of fluorescence structures as a vector field. This "fluorescence motion analysis" (FMA) uses optical flow techniques to analyze the movement and change of intensity gradients. This FMA approach is quite similar to the "gaseous image velocimetry (GIV)" technique introduced by Grünefeld et al. (2000a, b) and Krüger (2001) which in turn is based to a certain extent on the "image correlation velocimetry (ICV)" proposed by Tokumaru and Dimotakis (1995). However, in contrast to GIV (and ICV), the FMA approach uses a different evaluation technique (i.e., more suitable optical flow methods). Furthermore, FMA does not necessarily aim to measure the flow velocity field but the quantitative motion of structures (see below). For this reason, we decided not to use the misleading term "velocimetry" and called this technique "fluorescence motion analysis" (FMA).

For the validation of this fluorescence motion analysis technique, synthetic LIF image pairs with known motion field were generated with different signal-to-noise ratios. Using these synthetic images as "ground truth" it was possible to scrutinize image pre-processing and the optical flow algorithms. Furthermore, the comparison of the calculated motion field with the ground truth shows the reliability and problems of this evaluation method. Finally, simultaneous double-pulse LIF and PIV measurements were conducted. This comparison gives an impression on how good the FMA result matches the actual flow velocity field and where specific differences can be identified.

\section{Experimental setup for double-pulse LIF}

In order to build a comparable, inexpensive setup for time resolved LIF investigations, a standard PIV setup was modified to become a double-pulse LIF setup: Two frequency doubled Nd:YAG lasers (Surelite, Continuum) were each equipped with a second doubler crystal and a beam combiner for the resulting emission wavelength of $266 \mathrm{~nm}$. The light sheet was formed by a standard PIV light sheet optic (LaVision) but equipped with quartz lenses.

To generate mixing situations under controlled conditions, a heatable and pressure resistant flow chamber with a coaxial nozzle was used as the test object. The flow of each component and the exhaust were controlled using metering valves. Gas conditions were monitored and controlled by pressure gauges (MKS Baratron) and thermo couples. This flow chamber was equipped with 3 quartz windows to allow the light sheet to pass the test section and to observe the induced fluorescence perpendicular to the light sheet. The fluorescence was captured by a PCO dual-frame CCDcamera equipped with an adaptable fast image intensifier (intensified relay optics, IRO, LaVision). The intensity fluctuations of the laser were monitored by coupling a few percent of the laser-light in front of the test section onto a second CCD-camera with a fluorescence screen which served as an energy monitor. This signal was used to correct the measured fluorescence intensities for pulse-to-pulse fluctuations of the laser energy (cf. Scholz et al. 2006, 2007 for more details). The whole system was controlled like a PIV system using a control computer with commercial PIV 
software (Davis 6.2, LaVision). This double-pulse LIF system works at $266 \mathrm{~nm}$ excitation with up to $8 \mathrm{~mJ} / \mathrm{pulse}$ with a minimum $\Delta t$ of $0.8 \mu$ s between two images (corresponding to $1.25 \mathrm{MHz}$ ) but only with a repetition rate of $10 \mathrm{~Hz}$ from image pair to image pair. On the one hand, this is a very low repetition rate from image pair to image pair, so that each image pair only gives a snapshot of the dynamic process. Therefore, this system is best suited to measure cyclic events or triggerable situations in transient flows. On the other hand, compared to standard high-speed LIF setups, this "cost-saving" setup provides quite high pulse energies (and therefore high fluorescence signals) even at the short wavelength of $266 \mathrm{~nm}$ and a much smaller delay between the images of the image pair (corresponding up to 1.25 MHz where high-speed LIF systems provide only some $\mathrm{kHz}$ ). Therefore, the question as to which system is the best choice strongly depends on the measurement task and the available experimental and financial resources.

\section{Double-pulse FARLIF imaging}

In our recent work we could verify that the fluorescence of toluene as a tracer in isooctane as well as the fluorescence of a special near standard gasoline (Shell colorless gasoline) is directly proportional to the fuel/air ratio under certain conditions as pressure, temperature and mixture ratio (Scholz et al. 2006, 2007). The use of this property for measuring the mixture ratio of fuel and air (for example, in combustion engines) is known as FARLIF (fuel/air ratio measurement by Laser Induced Fluorescence) and was first introduced by Reboux et al. (1994).

For example, in the case of "colorless gasoline" as fuel, this FARLIF principle is applicable at pressures above 2.5 bar, with sufficient air-fraction $\lambda \geq 0.4$ and temperatures at least up to $550 \mathrm{~K}$ (Scholz et al. 2007). Figure 1 depicts this linearity between fluorescence intensity and equivalence ratio which is a measure of the fuel/air ratio. This means that planar LIF images can be calibrated to fuel/air ratio maps using calibration curves such as the one presented in Fig. 1. This approach is viable as long as laser attenuation due to absorption can be neglected [as discussed in Scholz et al. (2007)].

As an example, Fig. 2 shows an image pair captured by double-pulse FARLIF measurement of a rich colorless gasoline fuel pulse moving and mixing in the surrounding air at a temperature of $T=500 \mathrm{~K}$ and pressure between 5 and 6 bar. In this mixture situation the fuel valve was shortly opened and then closed again during constant coaxial air flow. The images were taken while closing the fuel-valve. The first image shows the head of the fuel pulse approximately $15 \mathrm{~mm}$ downstream of the nozzle and the highest equivalence ratio (or fuel/air ratio) near the nozzle exit,

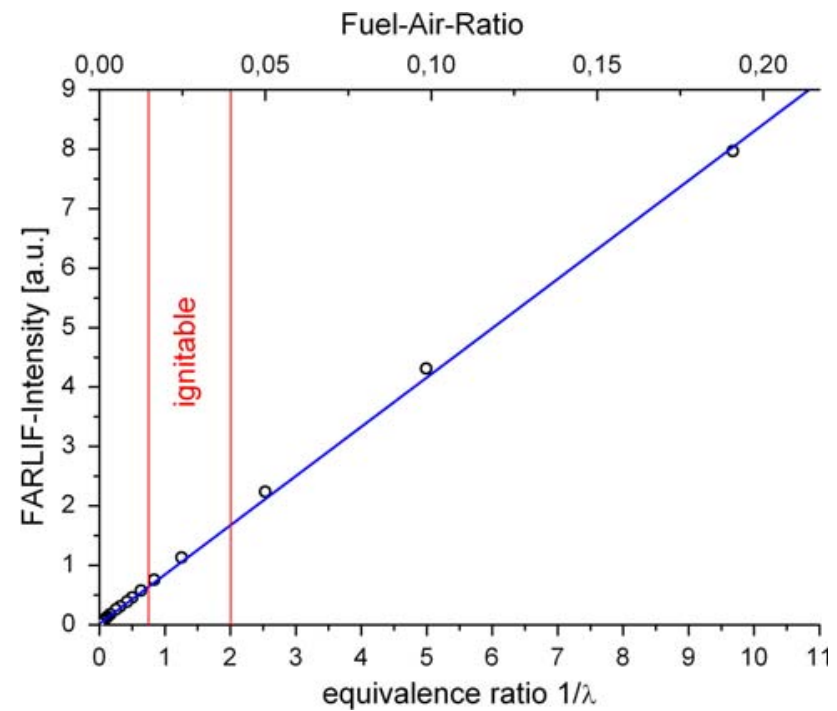

Fig. 1 FARLIF calibration curve: linearity between LIF intensity and fuel/air ratio (equivalence ratio, resp.)

indicating that the nozzle still emits fuel. The temporal delay between both images was $\Delta t=2.5 \mathrm{~ms}$ and the intensities have been calibrated to equivalence ratios (fuel/air ratios) using the corresponding calibration curve (as presented in Fig. 1). The comparison of both images show that the pulse front moves from bottom to top (compare the distance to the red dashed line at $16 \mathrm{~mm}$ which serves as a marker ${ }^{1}$ ), the shape stretches and the structure smears out due to mixing. Therefore, visually comparing a LIF double image renders at least a qualitative impression of the dynamics.

However, in some cases it is essential to find a more quantitative measure for the change between two images. One possibility is to determine the temporal derivative of the equivalence ratio. This is simply done by subtraction of the first image from the second (i.e., by subtraction of the intensity-value of each pixel in the first image from the intensity-value of the corresponding pixel in the second image) and by dividing the resulting image by the temporal delay between both images. The result is the two dimensional derivative field of the equivalence ratio distribution. Figure 3 shows the temporal derivative of the image pair from Fig. 2. The image is color-coded, showing areas with red denoting the strongest gain in equivalence ratio and green showing areas with strong loss in equivalence ratio as depicted by the color bar. The speckle-like noise in areas with small derivatives mainly stems from the shot-noise of the camera system at very high gain. It can be reduced by spatial filtering (not applied here). It is obvious that the biggest increase of the equivalence ratio is found at the top of the fuel-head. Here, the fuel-cloud moves with the gas

\footnotetext{
${ }^{1}$ Here the average displacement is about 6 pixels, the same order of magnitude as in PIV measurements.
} 
Fig. 2 Double-pulse FARLIFimage depicting the temporal evolution of fuel/air-ratio

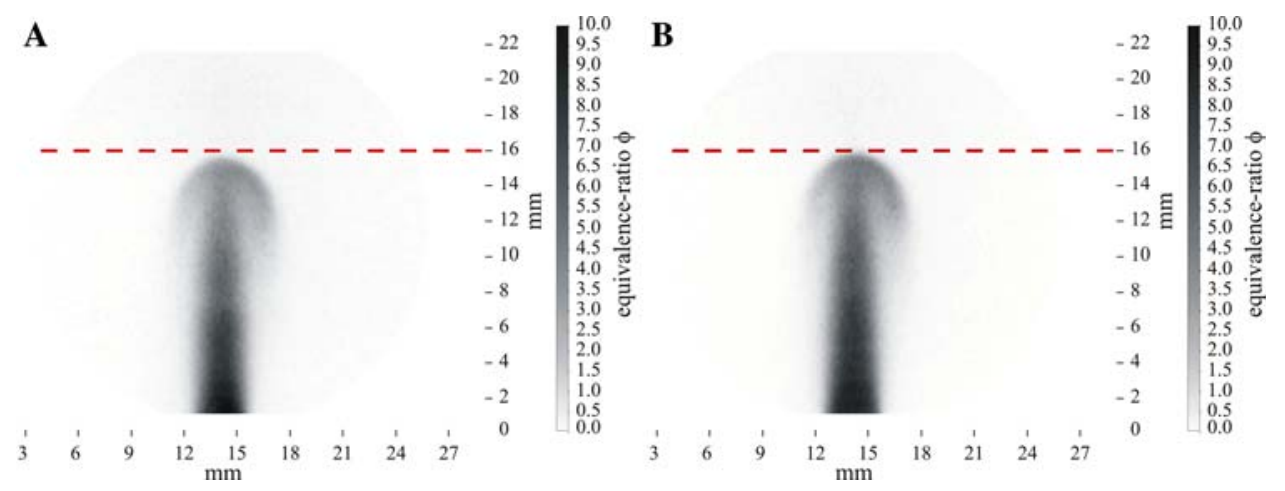

of the structures. As mentioned in Sect. 1, this approach is to a certain extent similar to the "gaseous image velocimetry (GIV)" of Grünefeld et al. (2000, b) and Krüger (2001) and the "correlation image velocimetry (CIV)" approach of Tokumaru and Dimotakis (1995). In contrast to those techniques, the approach presented in this paper does not aim to measure (necessarily) the flow velocity field but the quantitative motion of structures. Therefore, to differentiate our approach from previous ones, we avoid the often misleading term "velocimetry" and name this combination of double-pulse LIF imaging and motion analysis "fluorescence motion analysis (FMA)". Furthermore, compared to the precursor techniques, FMA uses different motion estimators for computing the optical flow field. The optical flow method that Grünefeld et al. and Tokumaru at al. employ, yields a highly non-convex optimization problem which may have many local minima (Krüger 2001; Scarano 2002; Tokumaru and Dimotakis 1995).

Krüger (2001) describes that much effort is needed to compute an initial guess for the solution in order to prevent the algorithm from being trapped in such a local minimum. The final solution will depend decisively on this initialization. Our approaches, in contrast, lead to a convex optimization problem. Its unique global optimum can be found in a reliable way by using standard techniques from convex optimization. The current state of the art estimators, both in terms of accuracy and applicability have been employed here. In the following, these state of the art estimators will be introduced briefly:

Variational methods for motion analysis go back to the early 1980s (Horn and Schunck 1981) and were originally developed for more general motion estimation tasks (motion in traffic scenes, robot vision, ...). Since then, there has been a great deal of research on different methods for the recovery of optical flow in different scenarios (e.g., Barron et al. 1994; Beauchemin and Barron 1995). This also led to the development of variational methods for the analysis of meteorological flows and fluid flows (Béréziat et al. 2000; Corpetti et al. 2002; Ruhnau et al. 2005); these methods form the basis of the presented approaches. 
The image data as visualized with the double-pulse FARLIF technique in this special application exhibit three characteristics:

1. only two successive images are available

2. the intensities between the two frames fluctuate due to changes in laser intensity

3. the images are corrupted by strong noise owing to the low signal intensities

Due to these limitations on the image data, variational optical flow techniques are methods of choice for computing highly accurate motion fields. While Haußecker et al. (1999) and Haußecker and Fleet (2001) presented techniques for estimating motion in the presence of general brightness models in a local structure tensor framework (Bigün et al. 1991), these approaches generally rely on the extraction of accurate spatio-temporal gradients form the images. Particularly in the presence of strong noise, structure tensor methods requires several successive images for recovering highly accurate gradients. For the present application, a local approach would thus lead to noisy motion fields or very sparse ones, if inaccurate flows are excluded by using confidence measures (confer Kondermann et al. 2007).

Two different approaches for dealing with the intensity fluctuations present in the images are feasible. These fluctuations can be modeled by a linear source term, or a constraint equation invariant to brightness changes can be used. In the following, these two approaches will be outlined and applied to the image data.

\subsection{Variational gradient based approach}

Let $I\left(x_{1}, x_{2}, t\right)$ denote the gray value recorded at location $\left(x_{1}, x_{2}\right)^{T}$ and time $t$ in the image plane. The basic assumption underlying most approaches to motion estimation is the conservation of $I$ over time:

$I\left(x_{1}+u_{1} \Delta t, x_{2}+u_{2} \Delta t, t+\Delta t\right)=I\left(x_{1}, x_{2}, t\right)$

This assumption is violated in the case of changing gray values due to, e.g., illumination changes. Let us therefore exchange (1) by

$I\left(x_{1}+u_{1} \Delta t, x_{2}+u_{2} \Delta t, t+\Delta t\right)=I\left(x_{1}, x_{2}, t\right)+b\left(x_{1}, x_{2}, t\right)$

where $b\left(x_{1}, x_{2}, t\right)$ is a scalar field that takes into account the above mentioned illumination changes. Note that the observed illumination changes arise from a multitude of effects (out-of-plane velocity, properties of the expanded laser beam, camera noise, ...). We have chosen this very simple (additive) term for modeling illumination/brightness changes, as the exact interaction of the different effects is usually not known and would require the use of many new parameters.
Let us take into account smooth changes of the flow $\left(u_{1}, u_{2}\right)^{T}$ at time $\mathrm{t}$ as a function of $x_{1}$ and $x_{2}: u_{1}=u_{1}\left(x_{1}, x_{2}\right)$, $u_{2}=u_{2}\left(x_{1}, x_{2}\right)$, and minimize

$$
\begin{aligned}
\int_{\Omega} & {\left[I\left(x_{1}+u_{1} \Delta t, x_{2}+u_{2} \Delta t, t+\Delta t\right)\right.} \\
& \left.-I\left(x_{1}, x_{2}, t\right)-b\left(x_{1}, x_{2}, t\right)\right]^{2} d x
\end{aligned}
$$

From the viewpoint of variational analysis and algorithm design, formulation (3) is less favorable because the dependency on $u_{1}$ and $u_{2}$ is highly non-convex. A common way around this difficulty is (1) to further simplify the objective function so as to obtain a mathematically tractable problem, and (2) to apply the resulting variational approach to a multi-scale representation of the image data I (cf, e.g., Ruhnau et al. 2005), so that the following approximation becomes valid:

$$
\begin{aligned}
& I\left(x_{1}+u_{1} \Delta t, x_{2}+u_{2} \Delta t, t+\Delta t\right) \\
& \quad \approx I\left(x_{1}, x_{2}, t\right)+\left(\partial_{x_{1}} I u_{1}+\partial_{x_{2}} I u_{2}+\partial_{t} I\right) \Delta t \\
& \quad=I\left(x_{1}, x_{2}, t\right)+\left(\nabla I \cdot\left(u_{1}, u_{2}\right)^{T}+\partial_{t} I\right) \Delta t
\end{aligned}
$$

where the spatial and temporal derivatives of $I$ can be estimated locally using FIR filters.

For a detailed description, we refer the interested reader to Ruhnau et al. (2005). Inserting this approximation into (1) (and dropping the argument $\left(x_{1}, x_{2}, t\right)$ for convenience) yields:

$\nabla I\left(x_{1}, x_{2}, t\right)\left(u_{1}, u_{2}\right)^{T}+\partial_{t} I=b$

Using (5) and (6), the objective function (3) becomes:

$\int_{\Omega}\left[\nabla I\left(x_{1}, x_{2}, t\right)\left(u_{1}, u_{2}\right)^{T}-\partial_{t} I-b\right]^{2} \mathrm{~d} x$

Note that this objective function now depends quadratically on the functions $u_{1}\left(x_{1}, x_{2}\right), u_{2}\left(x_{1}, x_{2}\right)$, and $b\left(x_{1}, x_{2}\right)$, which is much more convenient from the mathematical point-ofview. So far, the transition to a continuous setting has led us to formulation (7), which has to be minimized with respect to arbitrary functions $u_{1}, u_{2}$, and $b$. Clearly, this problem is not well-posed as yet because any vector field with components $\nabla I\left(x_{1}, x_{2}\right)^{T}-b=\partial_{t} I, \forall x_{1} ; x_{2}$, is a minimizer. Let us therefore rule out too irregular vector fields and brightness functions by additionally minimizing the magnitudes of the spatial gradients of $u_{1}, u_{2}$, and $b$ :

$$
\begin{aligned}
& J\left(u_{1}, u_{2}, b\right)=\int_{\Omega}\left[\nabla I\left(u_{1}, u_{2}\right)^{T}-\partial_{t} I-b\right]^{2} \\
& \quad+\lambda\left(\left|\nabla\left(u_{1}, u_{2}\right)^{T}\right|_{2}^{2}\right)+\mu|\nabla b|^{2} \mathrm{~d} x, 0<\lambda, \mu \in \mathcal{R}
\end{aligned}
$$

where $\lambda$ and $\mu$ are user-parameters. We discretize (8) by using standard first-order finite elements. The Gâteaux 
derivatives in the directions $u_{1}, u_{2}$ and $b$ yield the firstorder necessary conditions. This so-called optimality system is a linear system that can be solved for the unknowns $u_{1}, u_{2}$ and $b$ by some corresponding iterative solver (Hackbusch 1993). For regularization terms that are more enhanced (and more physically plausible) than this simple smoothness prior, we refer to Suter (1994), Corpetti et al. (2005), Ruhnau and Schnörr (2007) and Ruhnau et al. (2007). These terms theoretically make possible the reconstruction of even very high frequency components of the velocity field it is well-known, however, that they are rather noise sensitive and less robust than the simple firstorder regularization of (8). As we are dealing in this manuscript with extremely difficult image material (little texture and the presence of noise), we confine ourselves to the robust first-order regularization of (8).

Note that vector validation methods are obsolete in connection with our approach as the regularization term considers spatial context during minimization. Due to the filling-in effects (cf., e.g., Horn and Schunck 1981) of our regularizer, however, velocity vectors will even be computed at those locations at which no gradient information is present. We exclude these (uncertain) vectors in a postprocessing step that analyzes the amplitude of the signal and of its spatial gradient.

\subsection{Variational cross correlation approach}

A second approach relies on an invariant formulation (with respect to additive and multiplicative intensity variations) of the constraint equation. One such invariant is the cross correlation between image patches. The negative cross correlation $E_{c c}$ is defined as

$E_{c c}(I(x), u(x))=-\frac{\operatorname{cov}^{2}\left(I_{1}(x), I_{2}(x+u(x) \Delta t)\right)}{\operatorname{var}\left(I_{1}(x)\right) \operatorname{var}\left(I_{2}(x+u(x) \Delta t)\right)}$

where $I_{1}$ and $I_{2}$ denote the images at the times $t$ and $(t+$ $\Delta t$ ), respectively. The covariance and the variance of the image intensities in the domain $\Omega$ are denoted by cov and var.

In image registration, Hermosillo et al. (2002) formulated a local cross correlation framework along with more sophisticated statistical data terms in a variational framework. Following Hermosillo et al. (2002), the local cross correlation can be used as a data term similar to Eq. (6), resulting in

$J(u)=\int_{\Omega}\left[E_{c c}(I(x), u(x))+\lambda E_{r}(I(x), u(x))\right] \mathrm{d} x$

where $\Omega$ denotes the image domain, $E_{r}$ is the regularizer and $\lambda$ again a user parameter. In this formulation the same refined regularization terms mentioned previously can be

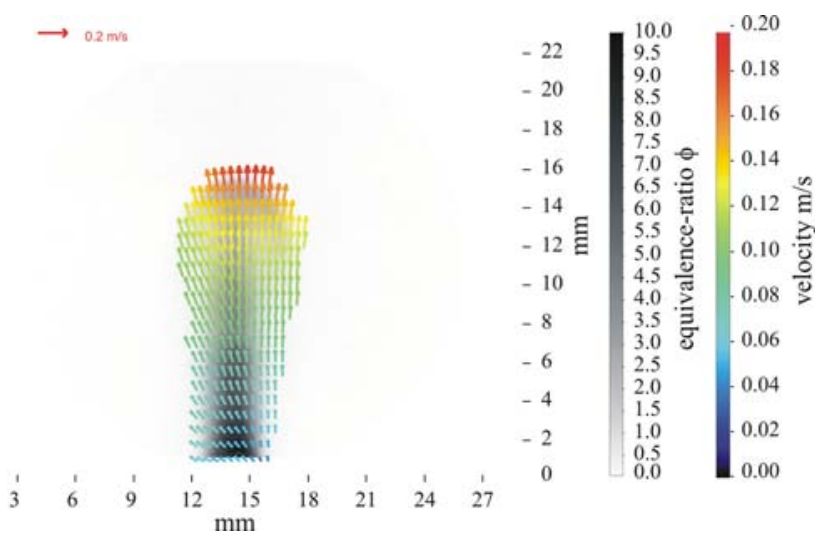

Fig. 4 Motion field of Fig. 2 evaluated by FMA

used. However, here we also limit ourselves to the robust first-order regularization for the same reason. The resulting Euler-Lagrange equations take the form

$$
\begin{aligned}
0= & \Delta u(x)-\frac{\operatorname{cov}\left(I_{1}, I_{2}\right)}{\operatorname{var}\left(I_{2}\right)}\left(\frac{I_{1}-<I_{1}>}{\operatorname{var}\left(I_{1}\right)}\right) \\
& -E_{c c}\left(\frac{I_{2}-<I_{2}>}{\operatorname{var}\left(I_{2}\right)}\right)
\end{aligned}
$$

where $\Delta$ denotes the Laplace operator and $\langle\cdot\rangle$ denotes the mean. It should be noted, that image warping with linear interpolation is being applied for all occurrences of $I_{2}$ in order to evaluate the image intensity at locations $(x+u(x) \Delta t)$.

\subsection{Application example for FMA}

Figure 4 shows the application of the variational gradient based approach on the image pair shown in Fig. 2. An example for the application of the variational cross correlation approach is given in Fig. 7 and will be discussed later. Figure 4 shows the motion of the intensity structures (i.e., the fuel cloud) of Fig. 2 as a vector field. On first sight, one essential feature of FMA results becomes obvious: the motion of the structures can naturally only be detected where strong enough structures occur and where structures (i.e., intensity gradients) are changing. It is an advantage of the variational gradient based approach, that the algorithm itself judges in a post-processing step where valid vectors can be detected. The vector field in Fig. 4 shows the fast movement of the pulse-head and the slower movement in its wake and at the nozzle exit. This is an expected observation as the corresponding double-pulse LIF measurement was conducted during the closing process of the fuel inlet ${ }^{2}$.

\footnotetext{
$\overline{2}$ The tendency of the flow to the left direction in Fig. 4 is caused by a slight asymmetrical installation of the nozzle.
} 
However, this does not mean that the motion field represents the actual flow velocity. This is exemplified in the homogeneous areas near the nozzle exit or homogeneous areas in the center of the cloud, where the flow velocity might differ from the motion of the structure (compare discussion in Sect. 6). Nevertheless, this example demonstrates the ability of the discussed FMA strategy to measure and visualize the movement of mixture structures quantitatively. Again, this technique may be translated to any other double-pulse LIF application (representing concentrations, phases, temperatures etc.) where structures are in motion

\section{Validation of FMA}

In order to obtain quantitative statements about the accuracy of the fluorescence motion analysis approach, the exact motion of the structures has to be known. One possibility to know the exact displacements is the simulation of the recording process of the LIF images. In such images the structures can be deformed and displaced arbitrarily. In order to yield results which are close to reality, the simulation of LIF images must be as realistic as possible. To fulfill this requirement, synthetic LIF images were generated as follows:

1. A real image (Fig. 5 left hand side) is used to define the regions which contain fluorescent structures by means of a mask (Fig. 5 middle).

2. In the regions defined by the mask, synthetic, Gaussian-shaped particle images are placed on an equidistant grid (one particle every 0.5 pixels). The particle image diameter $d_{P}$ is calculated according to $d_{P}=\operatorname{const} \sqrt{G}$ (with $G$ denoting the gray value) by means of a bilinear interpolation of the surrounding pixel grey values (cf. Fig. 6).

3. The synthetic first image with structure $\left(I_{1}\right.$, struc $)$ is calculated. This is done by using an in-house software
(Institute of Fluid Mechanics, TU Braunschweig) which was validated by means of the EUROPIV Synthetic Image Generator (Stanislas et al. 2004). The particle image shape is assumed to be Gaussian. Pixel gray values are added if one pixel is illuminated by several particles.

4. The particle images are displaced by applying a given equation. Thereafter, the second image with structure $\left(I_{2}\right.$, struc $)$ is calculated.

5. The synthetic images which have been generated so far only contain the (moving) LIF structure. In order to fill the background, two images containing only background and no LIF structure have been recorded beforehand ( $I_{1}$, back and $I_{2}$, back $)$ with the same experimental setup as the original LIF image. To combine $I_{1}$, struc and $I_{1}$, back an image $I_{1}$ is generated which inserts $I_{1}$, back where no LIF mask was defined. At the positions where the LIF mask is defined the gray value for $I_{1}$ is calculated by the following equation:

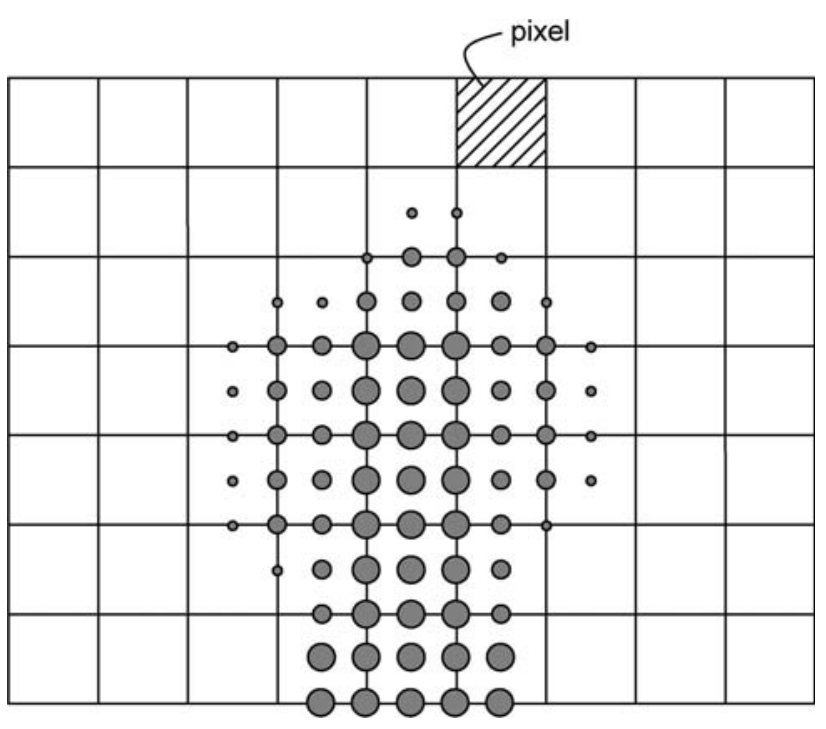

Fig. 6 Particle image distribution
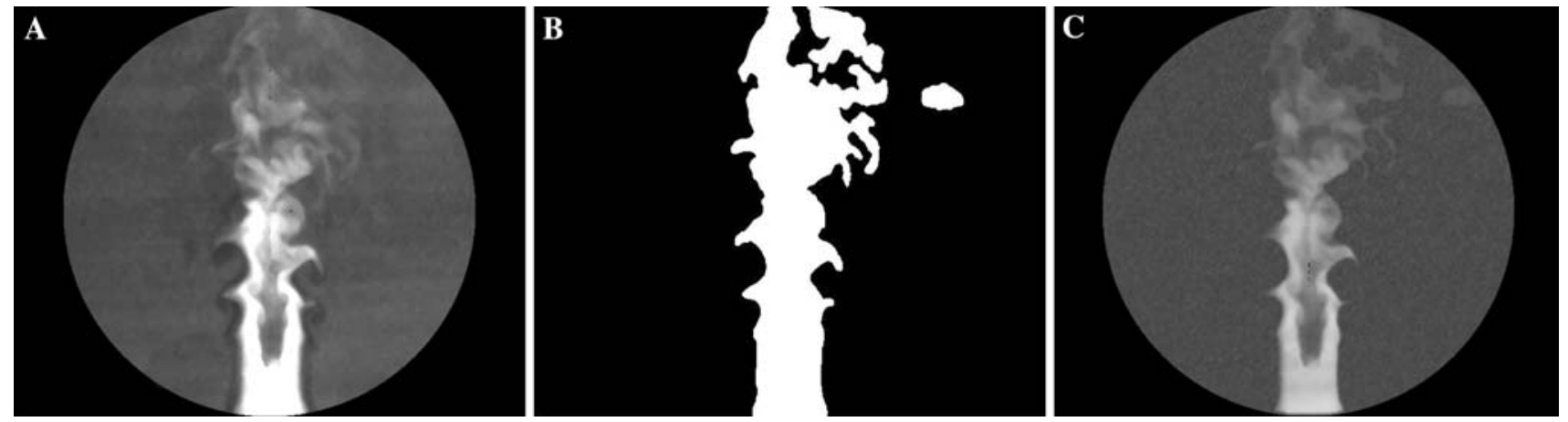

Fig. 5 Synthetic image generation: original image (left hand side), mask for the LIF structure (middle) and synthetic image with a background weighting of $40 \%$ (right hand side) 
$I_{1}(x, y)=W I_{1, \text { back }}(x, y)+(1-W) I_{1, \text { struc }}(x, y)$.

In order to obtain a continuous fading between structure and background, the weighting factor $W$ is linearly scaled between 1.0 and the prescribed value across the outer 5 pixels. A result with a background weighting of $40 \%$ is shown in Fig. 5 on the right hand side. $I_{2}$ is generated in a similar way.

The described method allows the generation of synthetic double-pulse LIF images which are close to reality. Hence, these images are well suited to assess the accuracy of evaluation methods like fluorescence motion analysis (FMA), which is applied in the following.

Figure 7 compares an original motion field (on the left) with the FMA result of the corresponding synthetic image pair, using the "variational cross correlation approach" described in Sect. 4.2 (on the right). The first image of the synthetic LIF image pair (as depicted in Fig. 5) is displayed in the background of each vector field. The simple structure of the motion field for this synthetic image pair was (to a certain extent) inspired by experimentally measured fields but has no real fluid dynamical background (e.g., is not a flow simulation), it just serves as the "ground truth" for the FMA evaluation. The comparison of the FMA result with the ground truth shows good agreement in most parts of the moving and distorting structure. Only in the upper areas, where the signal level is low and close to the noise level, differences in vector length (color) and direction become obvious. The relative deviation of the vector magnitudes from the ground truth is in the most parts below $10 \%$, but in areas with very week signal the deviation reaches a maximum of $70 \%$. The overall average deviation in this special case is $15 \%$.

The situation is even better, when the "variational gradient based approach" from Sect. 4.1 is applied for FMA evaluation. Figure 8 depicts a color-coded image of the relative deviation from the ground truth for different noise levels (background weighting $W$ in Eq. (12)). Without additional noise $(W=0)$ the deviation from the ground truth is in most areas far below 3\% (Fig. 8, left). Only at very weak structures such as the isle in the top right corner of the image the deviation reaches $15 \%$. The average deviation in this test case is $2.4 \%$. Here, in a comparison of vector fields (like Fig. 7) no differences could be seen by the eye. With $15 \%$ additional noise (Fig. 8 middle, $W=0.15$ ) the deviation rises but is still below $4 \%$ in most areas. Here, the maximum deviation is in the order of $20 \%$ and in this special case the average is $3.1 \%$. Even with a high additional noise level of $40 \%$ (Fig. 8 right, $W=0.4$ ) in most parts the deviation is below or in the order of $5 \%$ and the maximum deviation is in the order of $20 \%$. But here the advantage of this approach becomes obvious: the evaluation automatically judges where valid motions can be detected and therefore the resulting vector field
Fig. 7 Validation of fluorescence motion analysis using synthetic images. Left first image of a synthetic image pair with original motion field. Right FMA result using the variational cross correlation approach
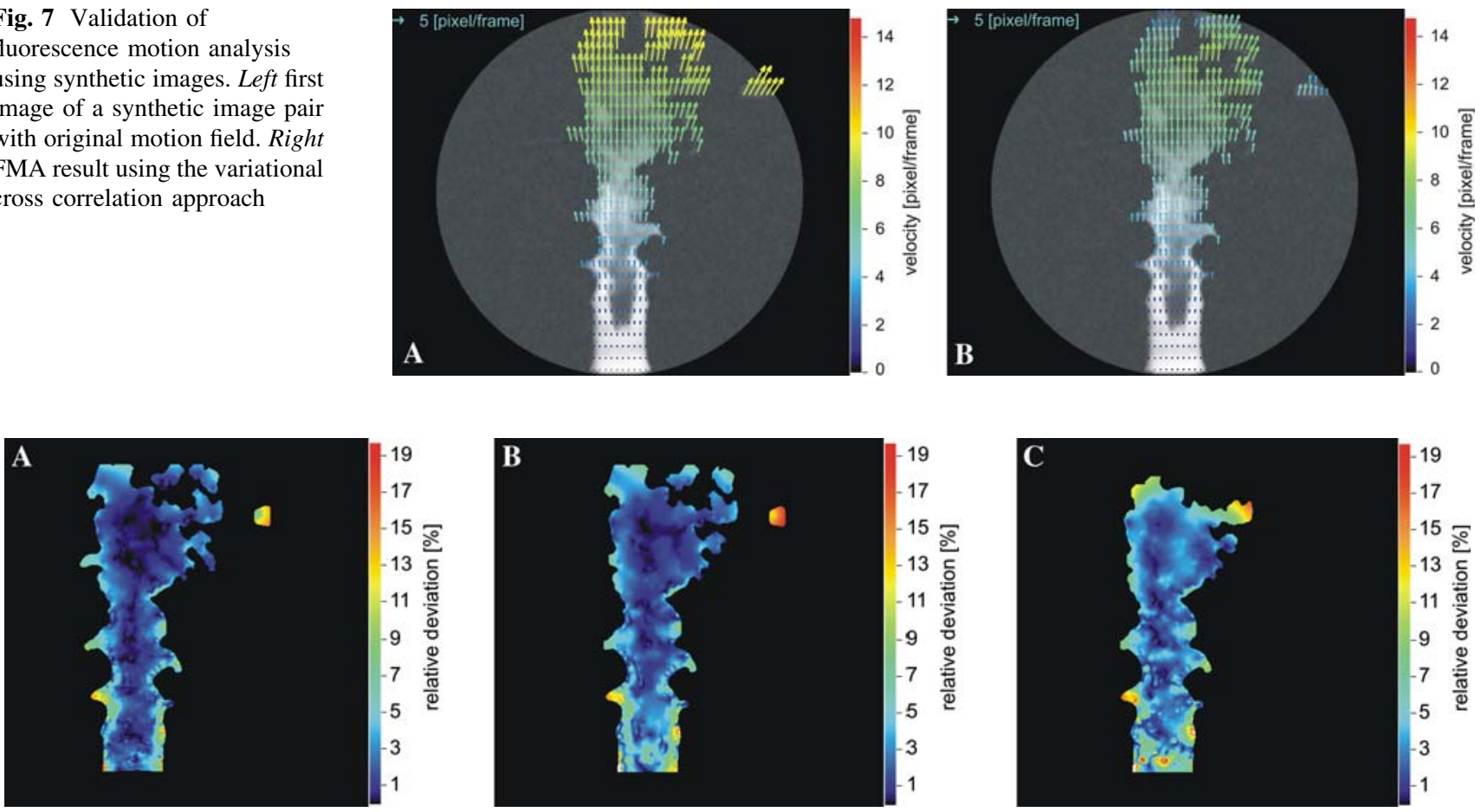

Fig. 8 Relative deviation from the original motion field for the variational gradient based FMA approach for different signal/noise levels. Left without noise; Middle 15\% noise; Right $40 \%$ noise 
(corresponding to the area where the deviation is calculated) is smaller. Even in this case of a very high noise level the average deviation is only $4.0 \%$.

These results show that FMA yields reliable estimates even under noisy conditions, whereas the "variational gradient based approach" seems to be preferable under the examined conditions. However, as the authors see much potential in the "variational cross correlation approach" as well, both approaches will be further investiageted in ongoing work.

\section{Experimental combination of double-pulse FARLIF with FMA and PIV}

As mentioned-above, fluorescence motion analysis not necessarily measures the whole and real flow field. Instead, FMA measures the structure motion and only gives results where structures are present and where they are changing. Yet, the flow field drives the mixture formation dynamics. Therefore, it is helpful to combine the above described double-pulse FARLIF technique with a standard particle image velocimetry (PIV) technique to gain deeper insight into the dynamic process. Furthermore, the combination of both techniques is able to reveal the differences of the corresponding results-which are vector fields in both cases.

In order to have exactly identical detection areas and detection time, the same double pulse laser light sheet at $266 \mathrm{~nm}$ was used for PIV and for FARLIF (therefore, no chromatic aberrations can occur, which might be possible if the second harmonic of the laser $(532 \mathrm{~nm})$ would be used for PIV simultaneously to the fourth harmonic $(266 \mathrm{~nm})$ for LIF, as another possibility for simultaneous PIV plus LIF which would be more common). A second double frame camera equipped with an image intensifier (IRO, to make the camera UV sensitive) was used for PIV detection at $266 \mathrm{~nm}$ and the splitting-up between PIV-signal and FARLIF-signal was achieved by reflection filters. It was important that the seeding-particles do not fluoresce.
Intense preliminary studies showed that an aerosol of pure "polyethyleneglycol 400" (PEG 400, purity: Ph Eur) is the best choice, with very low fluorescence but a good particle size with strong scatter-signal for PIV. The PIV measurements and evaluations were conducted with commercial PIV-software (DaVis 6.2, LaVision) using standard PIV algorithms with adaptive multi-pass, window shift and decreasing cell size.

Figure 9 presents an example of the results of a simultaneous single-shot PIV measurement and double-pulse FARLIF imaging with FMA evaluation in a transient mixing situation. The velocities are color-coded. The flow scenario is quite similar to the situation of Figs. 2, 3 and 4: during steady air flow the fuel valve was opened for a short time and then closed again. The gas temperature was $398 \mathrm{~K}$ and the total pressure about 5 bar. The result of double pulse FARLIF with fluorescence motion analysis using the "variational gradient based approach" is shown in the left part of Fig. 9. The right part depicts the flow field measured simultaneously by PIV. The background of each picture shows the calibrated fuel-air ratio field of the first FARLIF-image.

As expected, the first look at the two results shows the biggest difference: as the FMA evaluation only shows results within the structure (i.e., the rich fuel cloud), the PIV result covers the whole field. Similar to the situation of Fig. 4 the FMA result of Fig. 9 shows the highest velocities at the top of the fuel pulse head (green vectors). Further upstream near the nozzle the structure motion is slower due to the closing process of the fuel valve. Qualitatively, the situation is the same in the PIV velocity field and the order of magnitude of the corresponding velocities in both results match quite well. But in the case of the PIV result, the highest velocities appear in the center of the pulse head (red vectors) and these high velocities are not detected by FMA. The explanation why the highest flow velocities do not appear at the border of the pulse head is the deceleration of the pulse by the slower surrounding air. Therefore, the border moves slower (velocity comparable to the FMA result at the border: green vectors in both cases) and the
Fig. 9 Combination of simultaneous double-pulse FARLIF measurement with FMA and PIV measurement. Left motion field measured by FMA. Right flow velocity field measured by PIV
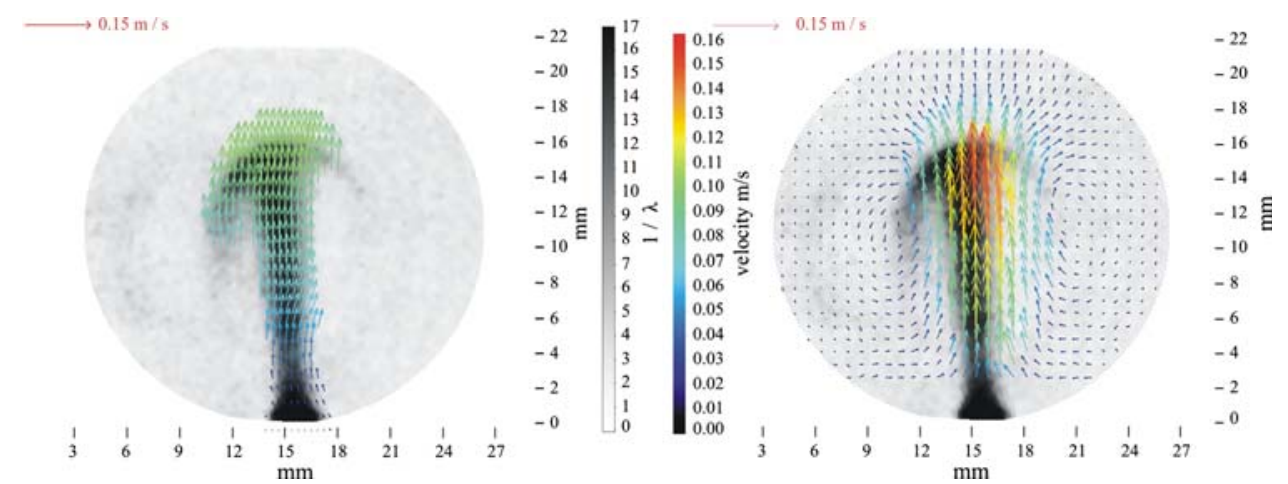
pulse forms its jellyfish-like shape. The reason why these high velocities inside the structure are not detected by FMA is the homogeneity of the structure in this area and the fact that here the flow velocity is directed parallel to the intensity structures. Therefore, the intensity structure locally does not change or move as fast. This example shows that in the case of homogeneous areas the FMA motion field naturally does not represent the velocity field (another example would be a laminar stationary coaxial flow, where structures do not change at all, although there is flow velocity). Homogeneous areas may also occur quite often in turbulent mixing scenarios: for example, about half of the mixed gas in a turbulent shear layer resides in more or less uniform vortex cores (Broadwell and Breidenthal 1982). Also in these cases the FMA result will not represent the velocity field in these homogeneous vortex cores. However, the example in Fig. 9 also shows that the FMA result provides different important information: at a glance, it represents the motion of the structure. Only from the PIV velocity field of Fig. 9 one would not expect such a slow structure motion. One would have to apply the velocity field on the structure (similar to the procedure in Sect. 5) to estimate its motion. Therefore, the presented FMA approach is very valuable in cases where the motion of fluorescence structures are important - as in the case of spark ignition engines where it is important if and when an ignitable cloud arrives at the spark plug. The technique can be applied in any case where the spatio-temporal evolution of the fluorescing property is of interest.

\section{Conclusions}

A concept for dynamic mixture formation analysis was presented using a converted standard PIV setup to perform double-pulse FARLIF imaging in the UV spectral range. Such a conversion of a PIV setup makes the presented measurement concepts affordable for many laboratories worldwide and is in some cases a cheaper and faster alternative to high-speed LIF setups. Double-pulse LIF images themselves give a first impression of the observed dynamic process. Furthermore, quantitative evaluation techniques for the double images give superior information density. As one possibility, the simple calculation of the temporal partial derivative of the intensity field - in the case of FARLIF the derivative of the equivalence ratio-was demonstrated. This evaluation shows where and how much the equivalence ratio is locally changing, giving a quantitative overview of the current mixture formation situation. The demonstrated approach may be transferred to any other LIF detection and gives insight into the change of the corresponding property detected by the LIF signal, like concentration, temperature, density etc.
As a key point of this paper, a second evaluation technique, the "fluorescence motion analysis (FMA)" was presented. This technique is able to quantitatively detect the motion of fluorescence structures such as moving clouds, distorting phases or mixing fluids. To a certain extent the technique borrows from precursor approaches "gaseous image velocimetry" and "correlation image velocimetry" but in contrast to those techniques, the approach presented in this paper does not aim to measure the flow velocity field but the quantitative motion of structures. Therefore, to differentiate our approach from previous ones, we avoid the often misleading term "velocimetry" and name this combination of double-pulse LIF imaging and motion analysis "fluorescence motion analysis (FMA)". Two different approaches for the motion estimation-the "variational gradient based approach" and the "variational correlation based approach"-were discussed and applied. Using synthetic LIF image pairs with a known motion field, it was possible to validate both approaches and to demonstrate their reliability and accuracy. The averaged relative deviation of the FMA result (variational gradient based approach) from the ground truth was only $2.5 \%$ for low noise signals and $4 \%$ under very noisy conditions where the deviations in most areas are far below these values in the specific test case. It seems that the "variational gradient based approach" is the better choice for the examined mixing scenarios but due to their potentials, both approaches will be pursued and improved in ongoing work. Again, the FMA approach may well be transferred to any other planar LIF detection scenario such as concentration-, temperature- or density-field imaging. Furthermore this approach may be used in the context of double-pulse Raman-imaging as well.

Additionally, the simultaneous application of the presented double-pulse FARLIF technique with FMA and standard PIV measurement was successfully demonstrated. The comparison of the FMA results with the simultaneously measured PIV flow velocity field showed that both results match quite well but represent different information: as the PIV result represents the flow velocity of the fluid which mainly drives the dynamical process, such as mixing, the FMA result represents the motion and distortion of the fluorescence structure. Both fields might not necessarily be the same as demonstrated in the given example of Sect. 6. However, both results, the velocity field and the structure motion are important in order to gain a deeper insight into the dynamic process; therefore a simultaneous measurement is the most valuable approach.

Finally, it has to be mentioned that all examined concepts and evaluation approaches in this paper can easily be transferred and adapted to various other planar LIF methods (or even planar Raman imaging) where the LIF signal represents, e.g., species concentration, temperature, density 
etc. - with the potential to broaden the insight for a wide range of dynamic processes.

Acknowledgments The authors gratefully acknowledge the financial support through the Deutsche Forschungsgemeinschaft DFG in the framework of the priority program SPP 1147.

\section{References}

Bigün J, Granlund GH, Wiklund J (1991) Multidimensional orientation estimation with application to texture analysis and optical flow. IEEE Trans Pattern Anal Mach Intell 13(8):775-790

Barron J, Fleet D, Beauchemin S (1994) Performance of optical ow techniques. Int J Comput Vis 39:43-77

Beauchemin SS, Barron JL (1995) The computation of optical flow. ACM Comput Surv 27(3):433-466

Béréziat D, Herlin I, Younes L (2000) A generalized optical flow constraint and its physical interpretation. In: CVPR, pp s2487s2492

Broadwell JE, Breidenthal RE (1982) A simple model of mixing and chemicalreaction in a turbulent shear layer. J Fluid Mech 125:397-410

Corpetti Th, Mémin E, Pérez P (2002) Dense estimation of fluid flows. IEEE Trans Pattern Anal Mach Intell 24(3):365-380

Corpetti Th, Heitz D, Arroyo G, Mémin E, Santa-Cruz A (2005) Fluid experimental flow estimation based on an optical-flow scheme. Exp Fluids 40(1):80-97

Grünefeld G, Bartelheimer J, Finke H, Krüger S (2000a) Gas-phase velocity field measurements in sprays without particle seeding. Exp Fluids 29:238-246

Grünefeld G, Finke H, Bartelheimer J, Krüger S (2000b) Probing the velocity fields of gas and liquid phase simultaneously in a two phase flow. Exp Fluids 29:322-330

Hackbusch W (1993) Iterative solution of large sparse systems of equations. Applied Mathematical Sciences, vol 95. Springer, Heidelberg

Haußecker H, Garbe C, Spies H, Jähne B (1999) A total least squares framework for low-level analysis of dynamic scenes and processes. In: DAGM Proceedings. Springer, Bonn

Haußecker H, Fleet DJ (2001) Computing optical flow with physical models of brightness variation. PAMI 23(6):661-673
Hermosillo G, Chefd'hotel C, Faugeras O (2002) Variational methods for multimodal image matching. Int J Comput Vis 50(3):329343

Horn B, Schunck B (1981) Determining optical flow. Artif Intell 17:185-203

Kondermann C, Kondermann D, Jähne B, Garbe CS (2007) An adaptive confidence measure for optical flows based on linear subspace projections. In: DAGM Proceedings. Springer, Heidelberg

Krüger S (2001) Laser diagnostics in two phase flows. PhD thesis, Faculty of Physics, University of Bielefeld, Bielefeld

Reboux J, Puechberty D, Dionnet F (1994) A new approach of PLIF applied to fuel/air ratio measurement in the compression stroke of an optical SI engine. SAE technical paper series, No. 941988

Ruhnau P, Schnörr C (2007) Optical stokes flow estimation: an imaging based control approach. Exp Fluids 42(1):61-78

Ruhnau P, Kohlberger T, Nobach H, Schnörr C (2005) Variational optical flow estimation for particle image velocimetry. Exp Fluids 38:21-32

Ruhnau P, Stahl A, Schnörr C (2007) On-line variational estimation of dynamical fluid flows with physics-based spatio-temporal regularization. Meas Sci Technol 18(3):755-763

Scarano F (2002) Iterative image deformation methods in PIV. Meas Sci Technol 13:R1-R19

Scholz J, Röhl M, Wiersbinski T, Beushausen V (2006) Verification and application of fuel-air-ratio-LIF. In: 13th international symposium on applications of laser techniques to fluid mechanics, Lisbon, pp 26-29

Scholz J, Wiersbinski T, Beushausen V (2007) Planar fuel-air-ratioLIF with gasoline for dynamic mixture-formation investigations, SAE Paper 2007-01-0645

Smith JD, Sick V (2006) A multi-variable, high-speed imaging study of ignition instabilities in a spray-guided, direct-injected, sparkignition engine, SAE Paper 2006-01-1264

Stanislas M, Westerweel J, Kompenhans J (2004) The EUROPIV Synthetic Image Generator (S.I.G.), Particle Image Velocimetry: Recent Improvements. Springer, Heidelberg

Suter D (1994) Motion estimation and vector splines. In: Proceedings of conference on computer vision and pattern recognition, $\mathrm{pp}$ 939-942

Tokumaru PT, Dimotakis PE (1995) Image correlation velocimetry. Exp Fluids 19:1-15 